\title{
The NET is closing in on inflammation in gout
}

T he causative agents of gout are monosodium urate (MSU) crystals, which deposit in peripheral joints leading to accumulation of neutrophils and to an acute and painful inflammatory response. In most cases, despite MSU crystals remaining in joints in structures known as tophi, this acute attack resolves in a few days. Until now the mechanisms behind this rapid resolution of inflammation were unknown. A paper published in Nature Medicine, from researchers at the University of Erlangen, Germany, shows that MSU crystals induce neutrophils to undergo oxidative burst and form neutrophil extracellular traps (NETs), which aggregate and degrade neutrophil-derived proinflammatory mediators, thereby limiting inflammation.

Markus Hoffmann, one of the lead researchers on this paper, says, "To investigate why acute highly inflammatory gout attacks resolve spontaneously after a few days even though the inflammatory trigger is still present in situ we built a very simple model of an acute gout attack in vitro: we isolated neutrophil granulocytes and exposed them to MSU crystals."

MSU crystals have been shown previously to induce the formation of NETs by neutrophils. NETs are extranuclear meshes of chromatin fibres studded with enzymes and antimicrobial proteins from neutrophil granules, which form when neutrophils undergo the specialized type of oxidative cell death known as NETosis. Using in vitro NETosis assays, the authors showed that at high densities of neutrophils, similar to those occurring in inflamed tissues, MSU crystals induce aggregation of NETs, forming complexes that share characteristics with gouty tophi.

"What quite surprised us was that the more cells we put into the culture, the lower the cytokine concentrations in the supernatants turned out to be, which was the opposite of what we had expected," says Hoffmann. Further experiments showed that, at high neutrophil density,
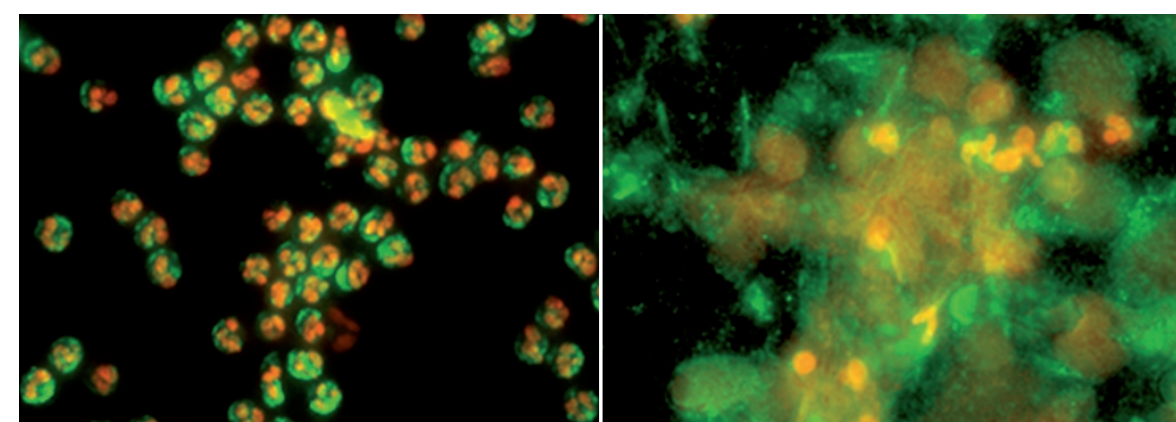

Human PMNs treated in vitro without (left) or with (right) MSU crystals, which at high cell concentrations results in the formation of aggregated NETs. Stained for DNA (DAPI) red, neutrophil elastase green, colocalization yellow. Image courtesy of Christine Schauer.

the neutrophil-derived chemokines and cytokines were not simply trapped inside the aggregated NETs, but were actively degraded by neutrophil serine proteases.

Alexander So (Centre Hospitalier Universitaire Vaudois, Switzerland), who was not involved in this study says, "The novel finding is that enzymes found within these NETs are capable of degrading inflammatory cytokines and chemokines, reducing their local concentrations and thereby turning off the recruitment of inflammatory cells." He adds that "NETs actually contribute to limiting the inflammatory process so that the gouty attack does not last forever."

The authors wanted to find out the in vivo implications of their findings so first they treated mice with thioglycolate (to cause accumulation of neutrophils in the peritoneum) and then injected MSU crystals intraperitoneally. Tophuslike aggregate structures comprising MSU crystals, NET-like structures and neutrophil-derived proteins were observed by dual energy computed tomography in these animals. These structures were not seen in mice that were not treated with MSU crystals.

Next the authors studied mice that cannot form NETs: $N c f 1^{\star *}$ mice, in which reactive oxygen species formation is blocked. They injected MSU crystals into air pouches of $N c f 1^{* *}$ and wildtype mice. Less aggregated NETs and higher levels of proinflammatory cytokines were seen in air pouches of MSU-treated $N c f 1^{\star *}$ mice than in MSU-treated wildtype mice. When aggregated NETs from wildtype mice were adoptively transferred into the air pouches of the MSU-treated $N c f 1^{* *}$ mice, levels of proinflammatory cytokines normalized.

Finally, wildtype and $N c f 1^{* *}$ mice were injected subcutaneously with MSU crystals. As expected, both sets of mice developed paw erythema and swelling; in the wildtype mice this acute inflammation resolved within 1-2 days, whereas in the $N c f 1^{* *}$ mice the inflammation was chronic and was evident for several weeks. These findings indicate for the first time that NETosis and NET aggregation leads to resolution of MSU-induced inflammation in gout.

"This research implies that modifying local concentrations of chemokines and cytokines may limit the severity of attacks of gout," states So. “This could be done by using enzymes that help to degrade local cytokines or by blocking cytokines directly; however the clinical relevance of these approaches needs to be tested."

Hoffman and colleagues plan to perform further research to see if targeting aggregated NETs could form the basis of a future therapeutic approach and to investigate if this mechanism is also of relevance for other neutrophil-mediated diseases.

Jenny Buckland

Original article Schauer, C. et al. Aggregated neutrophil extracellular traps limit inflammation by degrading cytokines and chemokines. Nat. Med. doi:10.1038/nm.3547 